\title{
Interventions to Reduce Unhealthy Alcohol Use among Primary Care Patients with HIV: the Health and Motivation Randomized Clinical Trial
}

\author{
Derek D. Satre, Ph.D. 1,2, Amy S. Leibowitz, Psy.D. ${ }^{2}$, Wendy Leyden, M.P. H $^{2}$, \\ Sheryl L. Catz, Ph.D. ${ }^{3}$, C. Bradley Hare, M.D. ${ }^{4}$, Hannah Jang, Ph.D. ${ }^{2}$, \\ Jennifer O. Lam, Ph.D. ${ }^{2}$, Kendall J. Bryant, Ph.D. ${ }^{5}$, Constance M. Weisner, Dr.P.H. , MSW ${ }^{1,2}$, \\ Stacy A. Sterling, Dr.P.H. ${ }^{2}$, Michael Horberg, M.D. ${ }^{6}$, Paul Volberding, M.D. ${ }^{7}$, and \\ Michael J. Silverberg, Ph.D., M.P.H. ${ }^{2}$
}

\begin{abstract}
'Department of Psychiatry, Weill Institute for Neurosciences, University of California, San Francisco, CA, USA; ${ }^{2}$ Division of Research, Kaiser Permanente Northern California, Oakland, CA, USA; ${ }^{3}$ Betty Irene Moore School of Nursing, University of California, Sacramento, CA, USA; ${ }^{4}$ Kaiser Permanente San Francisco Medical Center, San Francisco, CA, USA; ${ }^{5}$ National Institute on Alcohol Abuse and Alcoholism, Bethesda, MD, USA; ${ }^{6}$ Mid-Atlantic Permanente Research Institute, Kaiser Permanente Mid-Atlantic States, Rockville, MD, USA; ${ }^{7}$ AIDS Research Institute, University of California, San Francisco, CA, USA.
\end{abstract}

BACKGROUND: Unhealthy alcohol use has adverse effects on HIV treatment. Screening, brief intervention, and referral to treatment (SBIRT) has some evidence of efficacy but may not be sufficient for those with low motivation or comorbid substance use.

OBJECTIVE: To examine the effectiveness of motivational interviewing (MI) and emailed feedback (EF) among primary care HIV-positive patients, compared with treatment as usual care (UC) only, which included SBIRT.

DESIGN: Randomized clinical trial.

PARTICIPANTS: Six hundred fourteen adult HIV-positive patients in Kaiser Permanente Northern California who reported prior-year unhealthy alcohol use.

INTERVENTION: Participants were randomized to either three sessions of MI (one in person and two by phone), information regarding alcohol risks via EF through a patient portal, or UC alone. MI and EF participants who reported unhealthy alcohol use at 6 months were offered additional $\mathrm{MI}$ and EF treatment, respectively.

MAIN MEASURES: Participant-reported unhealthy alcohol use (defined as $\geq 4$ / $\geq 5$ drinks per day for women/ men), alcohol problems at 12 months, based on blinded telephone interviews. Secondary outcomes included drug use and antiretroviral (ART) adherence.

KEY RESULTS: At 12 months, there were no overall group differences, but in all three arms, there were declines in unhealthy alcohol use and alcohol-related problems $(p<0.001)$. Participants reporting low motivation to reduce drinking at baseline were less likely to report unhealthy alcohol use if they received MI vs. EF and UC ( $p=$ 0.013). At 6 months, reported illegal drug use/misuse of prescription drugs other than marijuana was lower in the MI arm than EF or UC ( $p=0.012)$. There were no differences in ART adherence between groups.

CONCLUSIONS: In a randomized trial of HIV-positive patients using two behavioral interventions compared with

Received October 15, 2018

Revised March 15, 2019

Accepted April 10, 2019

Published online June 11, 2019
SBIRT alone, participants in all three conditions reduced unhealthy alcohol use. MI may provide added benefit for patients with low motivation or who report illegal drug use/misuse of prescription drugs.

TRIAL REGISTRATION: NCTO1671501 (ClinicalTrials. gov)

KEY WORDS: HIV; alcohol; primary care; motivational interviewing; digital health; adaptive intervention; SBIRT.

J Gen Intern Med 34(10):2054-61

DOI: $10.1007 / \mathrm{s} 11606-019-05065-9$

(c) Society of General Internal Medicine 2019

\section{INTRODUCTION}

Reducing unhealthy alcohol use is a priority in primary care, especially for patients with HIV and other serious conditions. Prior studies have identified high rates of co-occurrence of HIV and unhealthy alcohol use, which puts individuals at risk for health problems. ${ }^{1-3}$ Drinking can compromise antiretroviral therapy $(\mathrm{ART})^{4,5}$ and retention in care, ${ }^{6}$ and increase rates of depression, ${ }^{7}$ HIV transmission risk behaviors, ${ }^{8}$ and mortality. ${ }^{9}$ Integrating brief interventions into primary care settings has been a major focus in health care, including efforts to deliver alcohol screening, brief intervention, and referral to treatment (SBIRT) at Kaiser Permanente Northern California (KPNC). ${ }^{10}$ SBIRT can be effective in reducing unhealthy alcohol use. ${ }^{11}$ However, patients with HIV, who often report other substance use in addition to alcohol, ${ }^{12}$ may require more extensive interventions. ${ }^{13}$ To address this gap, the Health and Motivation Study compared usual care with two promising interventions to reduce unhealthy alcohol use and comorbid drug use, provided as a supplement to systematic SBIRT, within a large HIV primary care clinic.

Motivational interviewing (MI) has been applied to many behavioral health problems, but most extensively tested for alcohol and drug use. ${ }^{14,15}$ Emailed feedback (EF) using 
secure messaging (between patient and physician) is based on research on computerized interventions including brief feedback regarding substance use risks. ${ }^{16,17} \mathrm{EF}$ has been used in prior research to deliver interventions to improve hypertension $^{18}$ and depression. ${ }^{19}$ In this study, we examined the efficacy of MI and EF in the context of HIV-positive patient care.

In this randomized trial, we hypothesized that both $\mathrm{MI}$ and EF would have a better effect on the main study outcomes of unhealthy alcohol use and alcohol-related problems relative to SBIRT alone, but that MI might be most effective due to its greater intensity and active components that enhance motivation. ${ }^{20}$ We anticipated a similar pattern of group effects on secondary outcomes of drug use, ART adherence, and HIV viral control. We also examined alcohol outcomes among nonalcohol-dependent enrollees, who have a greater likelihood of benefitting from brief interventions ${ }^{21,22}$, and among those who reported low motivation to reduce drinking at baseline.

\section{MATERIALS AND METHODS}

\section{Study Design and Setting}

The study was a randomized clinical trial, in which participants were assigned to MI, EF, or UC (1:1:1 allocation ratio). KPNC is a private non-profit integrated health system of 4 million members, covering over $40 \%$ of the region's insured population. Study participants were adults age 18 and older in the San Francisco KPNC Medical Center HIV Primary Care Clinic. The clinic provides treatment to about 3000 HIVpositive patients, following an HIV specialty model, with support provided by staff including nurses and clinical pharmacists. KPNC members have addiction treatment coverage and can access addiction clinics directly. ${ }^{23}$

\section{Participants}

Study inclusion criteria were based on self-report of any unhealthy alcohol use in the prior year. Because HIV-positive patients frequently are prescribed medications that can have adverse interactions with alcohol ${ }^{24}$ and may be more sensitive to alcohol effects, ${ }^{25}$ for eligibility we conservatively defined unhealthy alcohol use as any days consuming $\geq 3$ drinks in a day for women and $\geq 4$ drinks in a day for men in the prior 12 months. We examined drinking outcomes at this level as well as the level typically used for the general population (i.e., $\geq 4$ drinks in a day for women and $\geq 5$ drinks in a day for men). ${ }^{26}$ The only exclusion criterion was a recommendation from providers that a patient not be recruited due to medical or psychiatric acuity (which occurred 33 times). Details regarding the baseline sample have been published previously. ${ }^{27}$

\section{Measures}

Participant Interviews. Demographic data were obtained from participants at baseline. Alcohol and drug use questions included substance type (alcohol, cannabis, cocaine, amphetamines, hallucinogens, inhalants, sedatives other than as prescribed, opioids other than as prescribed, heroin or methadone, tobacco, and other) and drinking levels. For each substance, participants were asked the number of days of use in the past 30 days. The Addiction Severity Index ${ }^{28}$ was used to measure alcohol-related problems. The alcohol importance ruler and confidence ruler are 10-point scales asking participants to rate how important it is to cut down or stop drinking, ranging from 1 (not at all important) to 10 (extremely important), and how confident they are that they could cut down or stop if they wanted to. ${ }^{29}$ Similar to prior studies, we categorized responses to examine subgroups of participants based on importance level (1-3, low importance; 4-6, medium importance; and 7+, high importance). ${ }^{30,31}$

At each time point, participants were asked to report whether they were currently taking ART medication (yes/no); and "What is your best guess about how much of your prescribed HIV medications you have taken in the last month?" (dichotomized to $\geq 90 \%$ vs. $<90 \%$ ). Self-report ART adherence has been validated against other methods such as medication refills. ${ }^{32}$ Using this self-report adherence question allowed us to simultaneously measure alcohol use and adherence at the same point in time.

EHR Data. Screening for unhealthy alcohol use and brief intervention by providers is recorded in the EHR. Specialty addiction treatment initiation was examined using records of visits between enrollment and 12-month interview. HIV viral control was measured using data from the KPNC HIV registry. The registry is populated through electronic monitoring of inpatient, outpatient, and laboratory testing databases. We examined differences in HIV RNA levels using lab values closest to the dates of enrollment, 6-month and 12-month follow-up (either before or after these time points).

\section{Procedures}

Recruitment. Study procedures were approved by the University of California, San Francisco (UCSF) and KPNC Institutional Review Boards. Potential participants were identified via clinic patient lists, with HIV status confirmed through the HIV Registry. The clinic director sent a letter describing the study to all clinic patients and included a phone number to call if they wished not to be recruited. Participants were recruited by phone between April 25, 2013 and May 29, 2015. Research assistants telephoned all patients who did not opt out, screened them for eligibility using the alcohol use inclusion criterion, and invited eligible patients to complete a baseline interview in person at the clinic.

Enrollment. At the in-person baseline appointment, a research assistant obtained written informed consent. Enrolled 
participants used laptop computers to complete the baseline questionnaire. Participants were stratified based on alcohol dependence risk score. ${ }^{33}$ Standard randomization procedures for intervention studies were followed. ${ }^{34}$ An assistant with no other study involvement used a computerized randomization tool to generate assignments and placed them into sealed security envelopes given to the enrolling research assistant, who opened them in sequence.

Treatment Conditions. All participants received routine HIV primary care services as part of usual care (UC). Annual screening for unhealthy drinking based on NIHrecommended thresholds (any days of consuming $\geq 4 / \geq 5$ drinks in the prior 3 months for women/men respectively) was integrated into usual care in KPNC following the SBIRT model ${ }^{10}$ prior to the time that trial recruitment began (June 2013). Questions were asked by medical assistants following automated prompts and results were recorded in the EHR. Physicians advised those who screened positive to reduce or quit drinking and referred those with significant alcohol-related problems to KPNC specialty addiction services. UC did not include screening or intervention for drug use.

The MI intervention consisted of one 45-min in-person session at the clinic followed by two 20-min telephone sessions, similar to prior MI alcohol studies. ${ }^{35,36}$ Treatment guidelines were based on motivational interviewing. ${ }^{37} \mathrm{MI}$ is a short-term, directive, patient-centered style of counseling to help explore and resolve ambivalence. The research clinician encouraged participants to minimize unhealthy alcohol use, cannabis (if participants reported problems associated with use), illegal drugs, and misuse of prescription drugs. The intervention incorporated information about substance use risks for people with HIV (e.g., the potential for alcohol and drug use to interfere with ART adherence) (Table 1). MI sessions were delivered within 6 weeks of enrollment.

The EF intervention consisted of an email sent by the research clinician via the secure messaging function of KPNC's patient portal. Content included information about risks associated with exceeding light-to-moderate drinking, as well as risks of drug use. EF messages were personalized to include feedback about each participant's baseline drinking and other substance use, and recommendations for further action (e.g., direction to online resources ${ }^{38}$ and KPNC addiction treatment) (Table 2). Each email encouraged participants to respond if they had questions.

The study clinician delivering the MI and EF intervention was a licensed clinical psychologist (author A.L.) experienced in substance use counseling. The study clinician received 15 hours of instruction and supervised practice of three training patients in MI and EF procedures (with author D.D.S.), as in prior trials. ${ }^{39}$

This trial examined MI and EF interventions in an adaptive format (in which treatment is intensified for non-
Table 1 Motivational Interviewing (MI) Intervention for Primary Care Patients with HIV and Unhealthy Alcohol Use

Format

45-min in-person session, as soon as possible after study enrollment.

20-min phone "booster" session, approximately 10 days after in-person MI session.

20-min phone "booster" session, approximately 14 days after initial booster.

Motivational interviewing approach

Evocative, exploring patient's own reasons for change; empathic and nonjudgmental.

Collaborative, meeting patients at their current level of motivation/phase of change.

Directive, gently guiding the patient in discussion of alcohol use. Adaptations for HIV-positive patients

Open-ended questions to evoke patients' goals and values, particularly related to HIV care, ART adherence, and overall health.

Reflective listening, strategically emphasizing "change talk" such as patient-generated reasons to decrease drinking and other drug use.

Summarizing, e.g., reviewing pros and cons of patient's current drinking and drug use patterns.

Developing discrepancy between goals/values and current behaviors, especially potential discrepancies between current use patterns and goals such as improved energy and functioning.

Rolling with resistance, acknowledging positive aspects of drinking, such as use of alcohol in social situations and to reduce stress.

Supporting self-efficacy, noting patient's efforts and successes such as self-care, routine primary care visits, and antiretroviral medication adherence.

Offering information about alcohol use and HIV, e.g., impact on medical symptoms and ART adherence in an MI-consistent manner (ask what patient already knows, ask permission to give additional information, tell brief information, and ask for patient's thoughts/feelings in response to this new information).

Comorbid drug use and related problems explored as needed, using MI strategies.

responders) ${ }^{40-42}$ Participants who reported unhealthy alcohol use in the 30 days prior to the 6-month interview were offered two additional 20-min MI telephone sessions (MI arm) or were sent an additional message regarding alcohol use risks (EF arm). However, we also examined 6-month outcomes to

Table 2 Emailed Feedback (EF) Intervention for Primary Care Patients with HIV and Unhealthy Alcohol Use

\section{Format}

Secure email delivered via Kaiser Permanente (KP)'s patient portal. Emailed feedback approach

Educational, providing information about low-risk drinking cutoffs, describing risks associated with exceeding those cutoffs, and including a link to NIAAA's Tips for Cutting Down on Drinking.

Personalized, with content based on patient demographics (age, sex), self-reported patterns of alcohol use and alcohol-related problems, and self-rated importance and confidence to make a change. Comorbid drug use identified as presenting additional risks to health.

Directive, containing a clear recommendation to limit use of alcohol and other substances, to cut back or quit (if participant exceeded low-risk limits), or utilize self-help and/or KP specialty services (if participant reported substance-related problems).

Adaptations for HIV-positive patients

In addition to general information about risks associated with drinking, all EF messages contained the following statement:

Limiting one's drinking is especially important for people who have a chronic health condition such as HIV, because alcohol:

-Increases the risk for antiretroviral medication side effects

-Decreases the effectiveness of some medications

- Can lead to missed medication doses, which increases the risk of disease progression and drug-resistance

-Increases the risk of using other drugs, and combining substances that can be harmful when taken together

In recognition of the special risks for HIV-positive patients, participants were advised to limit their drinking to a relatively conservative limit of no more than 1-2 drinks per day. 
determine the effects of the initial treatment dose of MI and $\mathrm{EF}$ relative to UC alone.

MI fidelity monitoring was conducted using the MI Treatment Integrity code (MITI 3.1). ${ }^{43}$ A sub-sample of $32 \mathrm{MI}$ sessions (15\% of participants) was coded by an experienced MI trainer (author D.D.S.), using 20-min recorded segments. EF fidelity monitoring included periodic review by D.D.S. of message content for consistency.

Follow-up Interviews. Research assistants blinded to treatment condition contacted participants by telephone 6 and 12 months after enrollment for a 30-min interview. Patient reporting via telephone follow-up has been reliable in substance use studies. ${ }^{44}$ Participants received $\$ 50$ gift cards for the baseline and 6-month interviews and $\$ 100$ for the 12month interview. Twelve-month follow-up interviews were completed by July 1, 2016.

\section{Analyses}

We examined comparability of the MI, EF, and UC groups on demographics, substance use, and readiness to change drinking, using one-way ANOVA to compare means across three groups and Pearson's chi-square test at baseline. We calculated MITI subscale means to determine results of MI fidelity monitoring. Participants' receipt of provider-delivered alcohol screening, brief intervention, and KPNC addiction treatment initiation between enrollment and 12-month follow-up were examined by study arm using the chi-square test.

We used one-way ANOVA to compare means across three groups and Pearson's chi-square to examine treatment group differences at baseline and 6 and 12 months. We also examined alcohol use outcomes among participants who did not meet criteria for alcohol dependence at baseline and among those who reported low motivation to reduce drinking (importance scale) using the Kruskall-Wallace test, because of nonnormal distribution of Likert scale responses. We calculated effect sizes using Cohen's $h$ for proportions in the full sample.

To examine changes over time in alcohol use and alcoholrelated problems, we employed generalized estimating equation methods ${ }^{45}$ using PROC GENMOD in SAS (Version 9.3, Cary, NC) to account for within-individual correlation between repeated observations, using robust standard errors and an exchangeable working correlation structure. We estimated that with three repeated measures, power to detect a difference of $8 \%$ or more in predicted probability of unhealthy alcohol use between groups would have power of 0.82 with a minimum sample of 177 per group. ${ }^{45}$ Analyses were conducted using SAS Version 9.3 and Stata $12 .{ }^{46}$

\section{RESULTS}

We initiated telephone contact with 2876 patients and spoke directly with 1571 (54.7\%). Among the patients spoken with and screened, 775 (49.3\%) met inclusion criteria and of these, $614(79.2 \%)$ enrolled. Of the 614 participants, 582 (94.8\%) completed the 6-month follow-up and 583 (95.0\%) completed the 12-month follow-up (Fig. 1). Participants at baseline ranged in age from 21 to 74, with a mean of 49.4 (standard deviation $(\mathrm{SD})=11.0) ; 595(96.9 \%)$ were male; $385(62.7 \%)$ were non-Hispanic White, 56 (9.1\%) were non-Hispanic Black, 86 (14.0\%) were Hispanic, 27 (4.4\%) were other, and $60(9.8 \%)$ unknown race/ethnicity. Four hundred forty-five $(72.4 \%)$ were employed, $360(60.8 \%)$ reported income $>\$ 50,000$, and 269 (43.8\%) were married/partnered. There were no significant baseline differences between groups on demographic or HIV measures, alcohol, and other substance use measures (Table 3).

EHR data indicated that between enrollment and 12-month follow-up, SBIRT clinic-based screening for unhealthy alcohol use was completed for $72.1 \%$ of MI (137/190), $69.2 \%$ of EF (135/195), and $73.4 \%$ of UC (146/199) participants. Using data from the first recorded screening after enrollment, positive screens were recorded for $18.3 \%$ of MI (25/137), $21.5 \%$ of EF (29/135), and $25.3 \%$ of UC (37/146) participants. Among those screening positive, physician-delivered brief interventions were provided for $52.0 \%$ of MI (13/25), $79.3 \%$ of EF (23/29), and 70.3\% of UC (26/37) participants. Rates of addiction treatment initiation among participants who completed the 12-month interview were $3.2 \%$ for MI (6/190), $4.1 \%$ for EF (8/195), and 3.5\% for UC (7/199) (not shown).

To measure MI fidelity, mean MITI scores were obtained for evocation (4.4; $\mathrm{SD}=0.58)$, collaboration $(4.9 ; \mathrm{SD}=0.29)$, autonomy/support $(5.0 ; \mathrm{SD}=0.00)$, direction $(4.7 ; \mathrm{SD}=0.49)$ and empathy $(5.0 ; \mathrm{SD}=0.00)$, reflection to question ratio (2.8; $\mathrm{SD}=1.40)$, percent open-ended questions $(89.7 ; \mathrm{SD}=15.6)$, percent complex reflections (59.8; $\mathrm{SD}=14.8)$, and percent $\mathrm{MI}$ adherent (100\%). These scores were above competence level on each dimension and were indicative of high MI fidelity. Fidelity monitoring in the EF arm did not involve scoring, but messages were reviewed periodically to maintain consistency.

There were no significant differences between study arms at 12 months in report of any prior 30-day unhealthy alcohol use at the $\geq 4 / \geq 5$ level (45 [23.8\%] in MI, 49 [25.1\%] in EF, and $54[27.1 \%]$ in usual care, $p=0.75$, Table 4 ). Outcomes were similar at the $\geq 3 / \geq 4$ level (not shown). Within each arm, the decreasing linear trend was significant for unhealthy alcohol use at the $\geq 4 / \geq 5$ level ( $p<0.001$ for all three arms) and for alcohol-related problems $(p<0.001$ for all three arms). Other drug use (any substance use other than marijuana and/or misuse of prescription drugs) was significantly different at 6 months $(25[12.4 \%]$ in MI, 45 [22.1\%] in EF, and 48 [23.0\%] in UC, $p=0.012)$ but not at 12 months (31 [15.4\%] in MI, 46 [22.6\%] in EF, and 44 [21.1\%] in UC, $p=0.16$ ). Among individuals reporting low importance of alcohol use reduction at baseline, there were significant differences of unhealthy alcohol use at the $\geq 4 / \geq 5$ level at 12 months (10 of 118 [8.9\%] in MI, 17 of 104 [16.8\%] in EF, and 25 of 112 [23.6\%] in usual care, $p=0.013)$. On measures of ART 


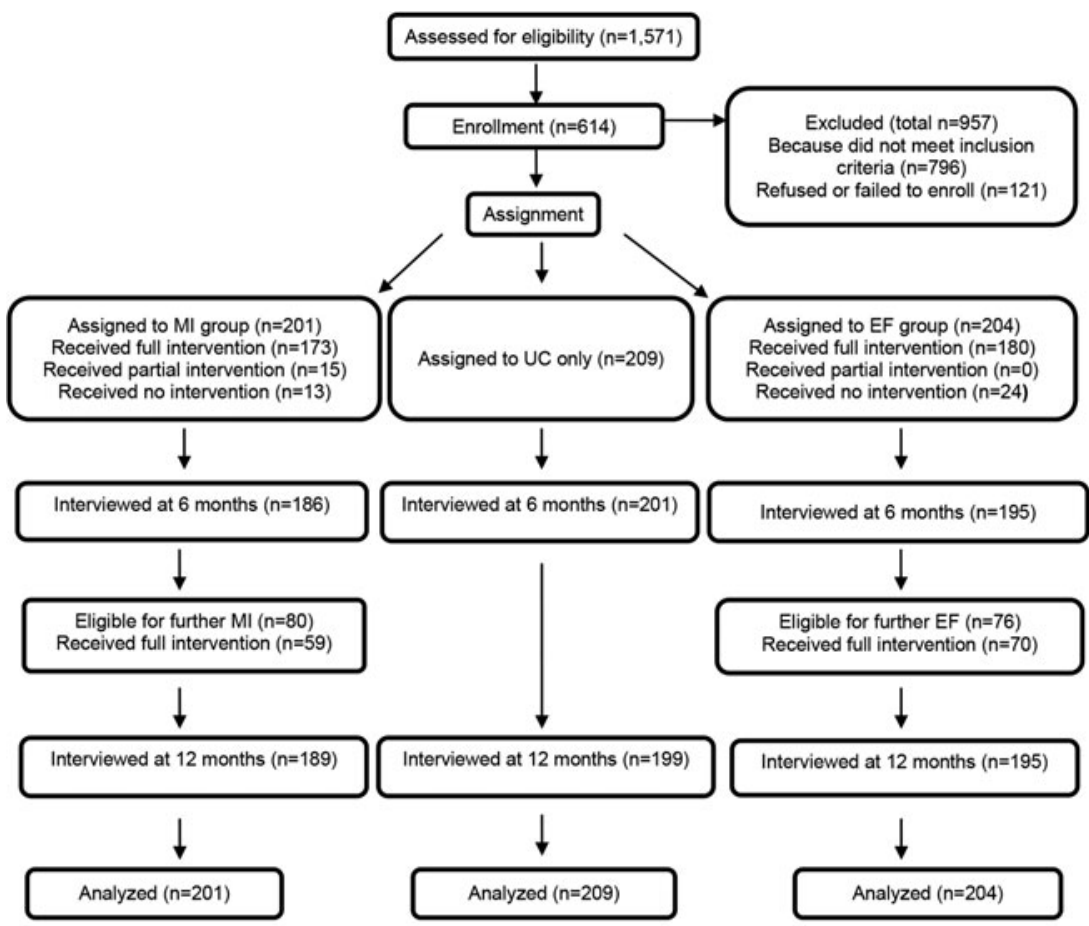

Note. This diagram is an adaptation of the flowchart offered by the CONSORT Group. MI = Motivational

Interviewing. $\mathrm{EF}=$ Emailed Feedback. All participants received usual care in the clinic. UC = usual care. Overall, 582 participants $(94.8 \%)$ completed the 6 -month telephone follow-up and 583 participants $(95.0 \%)$ completed the 12-month follow-up. Enrollment was conducted between 4/25/2013 and 5/29/2015, and follow-up interviews were completed by $7 / 1 / 2016$. No harms were reported. Non-receipt of full MI and EF study intervention was due to participant non-response to MI session scheduling requests (MI arm) and technology-related issues such as inactive secure messaging ( $E F$ arm). Losses to follow-up were due to participant non-response to interviewer phone calls. All enrolled participants were included in the outcome analyses.

Figure 1 Recruitment, assignment to treatment conditions, and follow-up of participants in the Health and Motivation Study.

Table 3 Baseline Cohort Characteristics of Participants by Intervention Treatment Arm $(N=614)$

\begin{tabular}{|c|c|c|c|c|}
\hline Characteristic & $\begin{array}{l}\text { Usual care } \\
(n=209)\end{array}$ & $\begin{array}{l}\text { Emailed feedback } \\
(n=204)\end{array}$ & $\begin{array}{l}\text { Motivational interviewing } \\
(n=201)\end{array}$ & $\overline{\chi^{2} p / \text { ANOVA }}$ \\
\hline Mean age $(\mathrm{SD})$, years & $49.9(10.9)$ & $49.0(11.4)$ & $49.2(10.7)$ & 0.65 \\
\hline Men, no. $(\%)$ & $205(98.1)$ & $197(96.6)$ & $193(96.0)$ & 0.46 \\
\hline Race/ethnicity, no. (\%) & & & & 0.68 \\
\hline Non-Hispanic White & $130(62.2)$ & $132(64.7)$ & $123(61.2)$ & \\
\hline Non-Hispanic Black & $18(8.6)$ & $18(8.8)$ & $20(10.0)$ & \\
\hline Hispanic & $27(12.9)$ & $24(11.8)$ & $35(17.4)$ & \\
\hline Other & $9(4.3)$ & $9(4.4)$ & $9(4.5)$ & \\
\hline Unknown & $25(12.0)$ & $21(10.3)$ & $14(7.0)$ & \\
\hline Education, no. (\%) & & & & 0.90 \\
\hline$\leq$ High school & $56(26.8)$ & $51(25.0)$ & $49(24.4)$ & \\
\hline Some college/college degree & $104(49.8)$ & $107(52.5)$ & $110(54.7)$ & \\
\hline Graduate school & $49(23.4)$ & $46(22.6)$ & $42(20.9)$ & \\
\hline Employed, no. (\%) & $144(68.9)$ & $148(72.6)$ & $153(76.1)$ & 0.26 \\
\hline Income $>\$ 50,000$, no. $(\%)$ & $122(60.1)$ & $115(58.4)$ & $123(64.1)$ & 0.50 \\
\hline Married/partnered, no. (\%) & $90(43.1)$ & $84(41.2)$ & $95(47.3)$ & 0.45 \\
\hline Years known HIV+, mean (SD) & $14.9(8.6)$ & $14.2(9.5)$ & $13.8(8.6)$ & 0.45 \\
\hline HIV exposure risk factor, no. (\%) & & & & 0.49 \\
\hline Men who have sex with men & $154(73.7)$ & $145(71.1)$ & $150(74.6)$ & \\
\hline Injection drug use & $14(6.7)$ & $12(5.9)$ & $13(6.5)$ & \\
\hline Heterosexual/other & $5(2.4)$ & $10(4.9)$ & $12(6.0)$ & \\
\hline Unknown & $36(17.2)$ & $37(18.1)$ & $26(12.9)$ & \\
\hline Any current tobacco use, no. (\%) & $47(22.5)$ & $56(27.5)$ & $47(23.4)$ & 0.46 \\
\hline High alcohol problem risk, no. (\%) & $118(56.5)$ & $87(57.4)$ & $116(57.7)$ & 0.97 \\
\hline DSM-IV alcohol dependent, no. (\%) & $54(25.8)$ & $50(24.5)$ & $47(23.4)$ & 0.85 \\
\hline Importance to reduce/stop drinking, mean (SD) & $4.0(3.1)$ & $4.1(3.1)$ & $3.7(2.9)$ & 0.29 \\
\hline Confidence to reduce/stop drinking, mean (SD) & $7.6(2.6)$ & $7.4(2.8)$ & $7.5(2.6)$ & 0.77 \\
\hline
\end{tabular}

$S D$, standard deviation. High alcohol problem risk is based on the two-item measure used for stratification. ${ }^{33}$ Importance and confidence were rated on a scale from 1 to 10, indicating low to high importance/confidence. Twenty-two participants did not answer the income question; percentages shown are among those who answered the question: $(122 / 203=60.1 \%, 115 / 197=58.4 \%, 123 / 192=64.1 \%)$ 
Table 4 Intervention Group Differences in Alcohol and Other Substance Use During Past 30 Days, ART Adherence and HIV Viral Suppression at Baseline, 6 and 12 Months. ( $N=614$ at baseline)

\begin{tabular}{|c|c|c|c|c|}
\hline Measure & $\begin{array}{l}\text { Usual care } \\
(n=209)\end{array}$ & $\begin{array}{l}\text { Emailed } \\
\text { feedback }(n=204)\end{array}$ & $\begin{array}{l}\text { Motivational } \\
\text { interviewing }(n=201)\end{array}$ & $\chi^{2} p /$ ANOVA \\
\hline \multicolumn{5}{|l|}{ Any drinking days of $\geq 4 / \geq 5$ drinks, no. (\%) } \\
\hline Baseline & $104(49.8)$ & $91(44.6)$ & $101(50.3)$ & 0.45 \\
\hline 6 months & $45(22.4)$ & $45(23.1)$ & $46(24.7)$ & 0.86 \\
\hline 12 months $^{\mathrm{a}}$ & $54(27.1)$ & $49(25.1)$ & $45(23.8)$ & 0.75 \\
\hline \multicolumn{5}{|c|}{ Number of drinking days of $\geq 4>5$ drinks, mean (SD) } \\
\hline Baseline & $3.5(6.6)$ & $2.2(4.3)$ & $3.4(6.6)$ & 0.06 \\
\hline 6 months & $1.2(3.5)$ & $1.1(3.2)$ & $1.5(4.2)$ & 0.58 \\
\hline 12 months & $1.4(3.9)$ & $1.3(3.5)$ & $1.6(4.6)$ & 0.80 \\
\hline \multicolumn{5}{|l|}{ Addiction Severity Index alcohol score (SD) } \\
\hline Baseline & $0.21(0.15)$ & $0.19(0.13)$ & $0.21(0.15)$ & 0.35 \\
\hline 6 months & $0.17(0.12)$ & $0.16(0.12)$ & $0.17(0.15)$ & 0.71 \\
\hline 12 months & $0.15(0.11)$ & $0.14(0.10)$ & $0.15(0.12)$ & 0.68 \\
\hline \multicolumn{5}{|l|}{ Any marijuana use, no. (\%) } \\
\hline Baseline & $106(50.7)$ & $101(49.5)$ & $99(49.3)$ & 0.95 \\
\hline 6 months & $83(41.3)$ & $83(42.6)$ & $75(40.3)$ & 0.91 \\
\hline 12 months & $88(44.2)$ & $84(43.1)$ & $81(42.9)$ & 0.96 \\
\hline \multicolumn{5}{|l|}{ Any other drug use, no. (\%) } \\
\hline Baseline & $59(28.2)$ & $57(27.9)$ & $52(25.9)$ & 0.84 \\
\hline 6 months*b & $48(23.0)$ & $45(22.1)$ & $25(12.4)$ & 0.01 \\
\hline 12 months $^{\mathrm{c}}$ & $44(21.1)$ & $46(22.6)$ & $31(15.4)$ & 0.16 \\
\hline \multicolumn{5}{|l|}{ Currently taking ART, no. (\%) } \\
\hline Baseline & $204(97.6)$ & $196(96.1)$ & $195(97.0)$ & 0.66 \\
\hline 6 months & $198(98.5)$ & $190(97.4)$ & $181(97.3)$ & 0.68 \\
\hline 12 months & $195(98.0)$ & $189(96.9)$ & $187(98.4)$ & 0.59 \\
\hline \multicolumn{5}{|c|}{$\geq 90 \%$ Adherence to ART past 30 days, self-report, no. (\%) } \\
\hline Baseline & $176(86.3)$ & $172(87.8)$ & $167(85.6)$ & 0.98 \\
\hline 6 months & $174(89.7)$ & $167(90.8)$ & $166(92.7)$ & 0.59 \\
\hline 12 months & $173(91.5)$ & $168(92.3)$ & $174(94.0)$ & 0.61 \\
\hline \multicolumn{5}{|l|}{ HIV RNA < 75 mL, no. (\%) } \\
\hline Baseline & 197 (94.7) & $185(91.7)$ & $189(94.0)$ & 0.41 \\
\hline 6 months & $194(97.0)$ & $179(92.3)$ & $173(93.0)$ & 0.10 \\
\hline 12 months & $192(96.5)$ & $181(92.8)$ & $177(93.1)$ & 0.23 \\
\hline $\begin{array}{l}\text { Participants not meeting alcohol dependence } \\
\text { criteria at baseline }\end{array}$ & $\begin{array}{l}\text { Usual care } \\
(n=155)\end{array}$ & $\begin{array}{l}\text { Emailed feedback } \\
(n=154)\end{array}$ & $\begin{array}{l}\text { Motivational interviewing } \\
(n=154)\end{array}$ & $\chi^{2} p /$ ANOVA \\
\hline \multicolumn{5}{|l|}{ Any drinking days of $\geq 4 / \geq 5$ drinks, no. (\%) } \\
\hline Baseline & $61(39.4)$ & $51(33.1)$ & $62(40.3)$ & 0.37 \\
\hline 6 months & 25 (16.9) & $22(14.8)$ & $28(19.1)$ & 0.62 \\
\hline 12 months & $29(19.7)$ & $24(16.2)$ & $19(12.9)$ & 0.29 \\
\hline $\begin{array}{l}\text { Participants who reported low importance to } \\
\text { reduce drinking at baseline }\end{array}$ & $\begin{array}{l}\text { Usual care } \\
(n=112)\end{array}$ & $\begin{array}{l}\text { Emailed feedback } \\
(n=104)\end{array}$ & $\begin{array}{l}\text { Motivational interviewing } \\
(n=118)\end{array}$ & Kruskall-Wallis test \\
\hline \multicolumn{5}{|l|}{ Any drinking days of $\geq 4 / \geq 5$ drinks, no. (\%) } \\
\hline Baseline & $42(37.5)$ & $38(36.5)$ & $48(40.7)$ & 0.80 \\
\hline 6 months & $17(15.7)$ & $16(15.8)$ & $18(16.2)$ & 0.99 \\
\hline 12 months $* \mathrm{~d}$ & $25(23.6)$ & $17(16.8)$ & $10(8.9)$ & 0.01 \\
\hline
\end{tabular}

"Other drug use" includes cocaine, heroin, ecstasy, hallucinogens, or prescription drug use other than prescribed. ART, antiretroviral therapy. "Low importance" to reduce alcohol $=$ score $<4$ on the importance ruler. $* p<0.05$

${ }^{a}$ For MI vs. UC, $h$ (effect size) $=0.08$ (confidence interval $[C I]=-0.12,0.28$ ); for $M I$ vs. EF, $h=0.03(C I=-0.17,0.23$ )

${ }^{b}$ For MI vs. UC, $h=0.28(C I=0.08,0.47)$; for $M I$ vs. $E F, h=0.26(C I=0.06,0.45)$

${ }^{c}$ For MI vs. UC, $h=0.15(C I=-0.05,0.34)$; for MI vs. $E F, h=0.18(C I=-0.01,0.38)$

${ }^{d}$ For MI vs. UC, $h=0.41(C I=0.14,0.68)$; for MI vs. $E F, h=0.24(C I=-0.03,0.51)$

adherence and viral control, there were no significant group differences (Table 4).

\section{DISCUSSION}

The study compared two adaptive interventions, a three-session MI intervention and a health plan patient portal-based EF intervention, with UC (SBIRT alone), in an alcohol-using primary care HIV-positive patient sample that also reported substantial rates of other substance use at baseline. Although there were no differences between groups in alcohol use outcomes, there was significant improvement within each group. The MI intervention was more effective at 12 months than the other treatment conditions in reducing drinking among those who reported lower motivation at baseline. Participants in the MI group also were less likely than others to report other drug use/ prescription drug misuse other than marijuana, but only at 6 months. These findings suggest that those with a more challenging clinical presentation associated with comorbid drug use $^{47}$ and low motivation could benefit from MI. ${ }^{48}$

Limited research has examined alcohol use interventions among HIV-positive patients, and outcomes to date have been mixed. ${ }^{49-51}$ In the current study, one possible explanation for 
the overall lack of significant differences was that all arms included clinician-delivered alcohol screening following the SBIRT model. Prior studies have found some evidence for alcohol SBIRT efficacy. ${ }^{11}$ Yet among individuals with HIV, brief interventions delivered by clinicians within the Veterans Affairs Health Care System were not associated with resolution of unhealthy alcohol use at 12 months. ${ }^{13}$ For individuals enrolled in a clinical trial and reporting a relatively high level of motivation to reduce drinking, it is possible that any of the study conditions could have been beneficial. Our finding of a significant intervention effect for MI among participants with lower motivation is consistent with the intent of this intervention, which is designed to enhance motivation for behavior change. ${ }^{37,52}$ Adaptive MI could be the most effective intervention approach for patients less motivated to reduce drinking, but implementation may require fidelity support ${ }^{53}$ or delivery by behavioral health specialists embedded in primary care. Given the lack of evidence for efficacy of providerdelivered SBIRT in reducing drug use, ${ }^{47}$ our findings regarding the effects of MI on drug use are promising and deserve further investigation in primary care.

\section{Study Limitations and Strengths}

The study was based in a single HIV primary care clinic in San Francisco and the sample was insured and primarily male, potentially limiting broad generalizability. However, demographic characteristics were similar to those of the clinic and the HIV-positive population in San Francisco, ${ }^{54}$ therefore maximizing internal validity and regional generalizability. UC did not include drug use screening or intervention, which may explain our finding of greater MI effectiveness in reducing drug use, and variability in the timing of SBIRT delivery relative to study follow-ups could have influenced outcomes. It was not possible to determine which participants read or engaged with material presented in the EF messages. All substance use measures were based on self-report and could be subject to social desirability or other reporting bias. Interview questions including alcohol-related problems and importance/confidence rulers may contribute to assessment reactivity. ${ }^{55}$ The study also had a number of strengths. We examined a topic of great clinical significance, unhealthy alcohol use, which has an adverse impact on treating HIV and other medical conditions. The study had a high enrollment rate, large sample, and high follow-up rates, and data included both interview- and EHR-based measures. The intervention arms encompassed two innovative experimental treatments with potential to be integrated into primary care, including a digital intervention, and examined the benefit of these treatments in addition to SBIRT alone.

\section{CONCLUSIONS}

This study examined the efficacy of two adaptive behavioral interventions to reduce unhealthy alcohol use, MI and EF, among primary care HIV-positive patients as a supplement to clinician-delivered SBIRT (UC). Participants in all three groups improved over 12 months. MI may provide added benefit for patients with low motivation to reduce drinking or who report both drinking and drug use.

Acknowledgments: We thank Stephen Follansbee, Alison Truman and Georgina Berrios for assistance in completing the study, and Agatha Hinman for assistance in preparing the manuscript.

Corresponding Author: Derek D. Satre, Ph.D.; Department of Psychiatry, Weill Institute for Neurosciences, University of California, Box 0984, San Francisco, 401 Parnassus Avenue, San Francisco, CA 94143, USA (e-mail: derek.satre@ucsf.edu).

Funding This study was funded by the National Institute on Alcohol Abuse and Alcoholism (UO1 AA021997 and K24 AA025703).

\section{Compliance with Ethical Standards:}

Conflict of Interest: All authors declare no conflicts of interest.

\section{REFERENCES}

1. Crane HM, McCaul ME, Chander G, Hutton H, Nance RM, Delaney JAC, et al. Prevalence and factors associated with hazardous alcohol use among persons living with HIV across the US in the current era of antiretroviral treatment. AIDS Behav 2017;21(7):1914-25.

2. Marshall BD, Operario D, Bryant KJ, Cook RL, Edelman EJ, Gaither JR, et al. Drinking trajectories among HIV-infected men who have sex with men: a cohort study of United States veterans. Drug Alcohol Depend 2015;148:69-76.

3. Saitz R. Clinical practice. Unhealthy alcohol use. N Engl J Med 2005;352(6):596-607.

4. Azar MM, Springer SA, Meyer JP, Altice FL. A systematic review of the impact of alcohol use disorders on HIV treatment outcomes, adherence to antiretroviral therapy and health care utilization. Drug Alcohol Depend 2010;112(3): 178-93.

5. Chander G, Lau B, Moore RD. Hazardous alcohol use: a risk factor for non-adherence and lack of suppression in HIV infection. $J$ Acquir Immune Defic Syndr 2006;43(4):411-7.

6. Monroe AK, Lau B, Mugavero MJ, Mathews WC, Mayer KH, Napravnik S, et al. Heavy alcohol use is associated with worse retention in HIV care. J Acquir Immune Defic Syndr 2016;73(4):419-25.

7. Sullivan LE, Goulet JL, Justice AC, Fiellin DA. Alcohol consumption and depressive symptoms over time: a longitudinal study of patients with and without HIV infection. Drug Alcohol Depend 2011;117(2-3):158-63.

8. Shuper PA, Joharchi N, Irving H, Rehm J. Alcohol as a correlate of unprotected sexual behavior among people living with HIV/AIDS: review and meta-analysis. AIDS Behav 2009;13(6):1021-36.

9. Fairbairn NS, Walley AY, Cheng DM, Quinn E, Bridden C, Chaisson C, et al. Mortality in HIV-infected alcohol and drug users in St. Petersburg, Russia. PLoS One 2016;11(11):e0166539.

10. Mertens JR, Chi FW, Weisner CM, Satre DD, Ross TB, Allen S, et al. Physician versus non-physician delivery of alcohol screening, brief intervention and referral to treatment in adult primary care: The ADVISe cluster randomized controlled implementation trial. Addict Sci Clin Pract 2015;10(26):26.

11. Babor TF, Del Boca F, Bray JW. Screening, Brief Intervention and Referral to Treatment: implications of SAMHSA's SBIRT initiative for substance abuse policy and practice. Addiction. 2017;112 Suppl 2:110-7.

12. DeLorenze GN, Satre DD, Quesenberry CP, Tsai AL, Weisner CM. Mortality after diagnosis of psychiatric disorders and co-occurring substance use disorders among HIV-infected patients. AIDS Patient Care STDs 2010;24(11):705-12.

13. Williams EC, Lapham GT, Bobb JF, Rubinsky AD, Catz SL, Shortreed SM, et al. Documented brief intervention not associated with resolution of unhealthy alcohol use one year later among VA patients living with HIV. J Subst Abus Treat 2017;78:8-14. 
14. Satre DD, Leibowitz A, Sterling SA, Lu Y, Travis A, Weisner C. A randomized clinical trial of Motivational Interviewing to reduce alcohol and drug use among patients with depression. J Consult Clin Psychol 2016;84(7):571-9.

15. Vasilaki EI, Hosier SG, Cox WM. The efficacy of motivational interviewing as a brief intervention for excessive drinking: A metaanalytic review. Alcohol Alcohol 2006;41(3):328-35.

16. Bendtsen $\mathbf{P}$, Johansson $\mathbf{K}$, Akerlind I. Feasibility of an email-based electronic screening and brief intervention (e-SBI) to college students in Sweden. Addict Behav 2006;31(5):777-87.

17. Schwartz RP, Gryczynski J, Mitchell SG, Gonzales A, Moseley A, Peterson TR, et al. Computerized versus in-person brief intervention for drug misuse: a randomized clinical trial. Addiction. 2014;109(7):1091-8.

18. Green BB, Cook AJ, Ralston JD, Fishman PA, Catz SL, Carlson J, et al. Effectiveness of home blood pressure monitoring, Web communication, and pharmacist care on hypertension control: a randomized controlled trial. JAMA. 2008;299(24):2857-67.

19. Simon GE, Ralston JD, Savarino J, Pabiniak C, Wentzel C, Operskalski BH. Randomized trial of depression follow-up care by online messaging. J Gen Intern Med 2011;26(7):698-704.

20. Kuerbis A, Armeli S, Muench F, Morgenstern J. Profiles of confidence and commitment to change as predictors of moderated drinking: a person-centered approach. Psychol Addict Behav 2014;28(4): 1065-76.

21. McCambridge J, Saitz R. Rethinking brief interventions for alcohol in general practice. BMJ. 2017;356:j116.

22. Jonas DE, Garbutt JC, Amick HR, Brown JM, Brownley KA, Counci $\mathrm{CL}$, et al. Behavioral counseling after screening for alcohol misuse in primary care: a systematic review and meta-analysis for the U.S. Preventive Services Task Force. Ann Intern Med 2012;157(9):645-54.

23. Satre DD, Mertens JR, Arean PA, Weisner C. Five-year alcohol and drug treatment outcomes of older adults versus middle-aged and younger adults in a managed care program. Addiction. 2004;99(10):1286-97.

24. Edelman EJ, Gordon KS, Glover J, McNicholl IR, Fiellin DA, Justice AC. The next therapeutic challenge in HIV: polypharmacy. Drugs Aging 2013;30(8):613-28.

25. Justice AC, McGinnis KA, Tate JP, Braithwaite RS, Bryant KJ, Cook RL, et al. Risk of mortality and physiologic injury evident with lower alcohol exposure among HIV infected compared with uninfected men. Drug Alcohol Depend 2016;161:95-103.

26. National Institute on Alcohol Abuse and Alcoholism. Helping patients who drink too much: a clinician's guide, updated 2005 edition. Rockville: U.S. Department of Health and Human Services 2005 [NIH Publication No. 07-3769], updated Jan 2007. http://pubs.niaaa.nih.gov/publications/Practitioner/CliniciansGuide2005/clinicians guide.htm Accessed March 15, 2019.

27. Silverberg MJ, Leyden WA, Leibowitz A, Hare CB, Jang HJ, Sterling S, et al. Factors associated with hazardous alcohol use and motivation to reduce drinking among HIV primary care patients: Baseline findings from the Health \& Motivation study. Addict Behav 2018;84:110-7.

28. McLellan AT, Kushner H, Metzger D, Peters R, Smith I, Grissom G, et al. The Fifth Edition of the Addiction Severity Index. J Subst Abus Treat 1992;9(3): 199-213.

29. Heather N, Smailes D, Cassidy P. Development of a Readiness Ruler for use with alcohol brief interventions. Drug Alcohol Depend 2008;98(3):235-40.

30. Bertholet N, Gaume J, Faouzi M, Gmel G, Daeppen JB. Predictive value of readiness, importance, and confidence in ability to change drinking and smoking. BMC Public Health 2012;12:708.

31. Hesse M. The Readiness Ruler as a measure of readiness to change polydrug use in drug abusers. Harm Reduct $\mathrm{J}$ 2006;3:3.

32. Simoni JM, Huh D, Wang Y, Wilson IB, Reynolds NR, Remien RH, et al. The validity of self-reported medication adherence as an outcome in clinical trials of adherence-promotion interventions: Findings from the MACH14 study. AIDS Behav 2014;18(12):2285-90.

33. Vinson DC, Kruse RL, Seale JP. Simplifying alcohol assessment: two questions to identify alcohol use disorders. Alcohol Clin Exp Res 2007;31(8):1392-8.

34. Hulley SB, Newman TB, Cummings SR, Hearst N, Grady D, Browner WS. Designing Clinical Research: An Epidemiologic Approach. 2nd ed. Philadelphia, PA: Lippincott Williams \& Wilkins; 2001.

35. Fleming MF, Balousek SL, Grossberg PM, Mundt MP, Brown D, Wiegel JR, et al. Brief physician advice for heavy drinking college students: a randomized controlled trial in college health clinics. J Stud Alcohol Drugs 2010;71(1):23-31.

36. Satre DD, Delucchi K, Lichtmacher J, Sterling SA, Weisner C. Motivational interviewing to reduce hazardous drinking and drug use among depression patients. J Subst Abus Treat 2013;44(3):323-9.

37. Miller WR, Rollnick S. Motivational Interviewing: Helping People Change. 3rd ed. New York: Guilford Press; 2013.

38. National Institute on Alcohol Abuse and Alcoholism. Tips for cutting down on drinking. Bethesda, MD: National Institute on Alcohol Abuse and Alcoholism 2007. Report No.: NIH Publication No. 07-3769.

39. Dunn C, Deroo L, Rivara FP. The use of brief interventions adapted from motivational interviewing across behavioral domains: a systematic review. Addiction. 2001;96(12):1725-42.

40. Edelman EJ, Maisto SA, Hansen NB, Cutter CJ, Dziura J, Fiellin LE, et al. The Starting Treatment for Ethanol in Primary care Trials (STEP Trials): Protocol for three parallel multi-site stepped care effectiveness studies for unhealthy alcohol use in HIV-positive patients. Contemp Clin Trials 2017;52:80-90.

41. Jaehne A, Loess1 B, Frick K, Berner M, Hulse G, Balmford J. The efficacy of stepped care models involving psychosocial treatment of alcohol use disorders and nicotine dependence: a systematic review of the literature. Curr Drug Abuse Rev 2012.

42. Drummond C, Coulton S, James D, Godfrey C, Parrott S, Baxter J, et al. Effectiveness and cost-effectiveness of a stepped care intervention for alcohol use disorders in primary care: pilot study. Br J Psychiatry 2009; 195(5):448-56

43. Moyers TB, Martin T, Manuel JK, Miller WR, Ernst D. Revised Global Scales: Motivational Interviewing Treatment Integrity 3.1.1 (MITI 3.1.1). University of New Mexico: Center on Alcoholism, Substance Abuse and Addictions (CASAA), 2010.

44. Cohen BB, Vinson DC. Retrospective self-report of alcohol consumption: Test-retest reliability by telephone. Alcohol Clin Exp Res 1995; 19(5): 1156-61.

45. Diggle PJ, Heagerty P, Liang K-Y, Zeger SL. Analysis of Longitudinal Data. 2nd ed. New York: Oxford University Press; 2002.

46. StataCorp. Stata Statistical Software: Release 12. College Station: StataCorp LP; 2011.

47. Saitz R, Palfai TP, Cheng DM, Alford DP, Bernstein JA, LloydTravaglini CA, et al. Screening and brief intervention for drug use in primary care: the ASPIRE randomized clinical trial. JAMA. 2014;312(5):502-13.

48. Aharonovich E, Stohl M, Ellis J, Amrhein P, Hasin D. Commitment strength, alcohol dependence and HealthCall participation: effects on drinking reduction in HIV patients. Drug Alcohol Depend 2014;135:1128 .

49. Williams EC, Hahn JA, Saitz R, Bryant K, Lira MC, Samet JH. Alcohol use and Human Immunodeficiency Virus (HIV) infection: current knowledge, implications, and future directions. Alcohol Clin Exp Res 2016;40(10):2056-72.

50. Scott-Sheldon LAJ, Carey KB, Johnson BT, Carey MP, Team MR. Behavioral interventions targeting alcohol use among people living with HIV/AIDS: A systematic review and meta-analysis. AIDS Behav 2017;21(Suppl 2):126-43.

51. Korthuis PT, Lum PJ, Vergara-Rodriguez P, Ahamad K, Wood E, Kunkel LE, et al. Feasibility and safety of extended-release naltrexone treatment of opioid and alcohol use disorder in HIV clinics: a pilot/ feasibility randomized trial. Addiction. 2017;112(6):1036-44.

52. Kahler CW, Pantalone DW, Mastroleo NR, Liu T, Bove G, Ramratnam B, et al. Motivational interviewing with personalized feedback to reduce alcohol use in HIV-infected men who have sex with men: A randomized controlled trial. J Consult Clin Psychol 2018;86(8):645-56.

53. Weisner C, Satre DD. A key challenge for motivational interviewing: training in clinical practice. Addiction. 2016;111(7):1154-6.

54. Das M, Chu PL, Santos GM, Scheer S, Vittinghoff E, McFarland W, et al. Decreases in community viral load are accompanied by reductions in new HIV infections in San Francisco. PLoS One 2010;5(6):e11068.

55. Meier E, Miller MB, Lombardi N, Leffingwell T. Assessment reactivity: A randomized controlled trial of alcohol-specific measures on alcoholrelated behaviors. Addict Behav 2017;67:44-8

Publisher's Note Springer Nature remains neutral with regard to jurisdictional claims in published maps and institutional affiliations. 